\title{
Editorial: Supporting Wellbeing During and After COVID-19 in Cities
}

\author{
Ruoyu Wang ${ }^{1 *}$, Linchuan Yang $^{2}$ and Hongsheng Chen $^{3}$ \\ ${ }^{1}$ School of GeoSciences, Institute of Geography, University of Edinburgh, Edinburgh, United Kingdom, ${ }^{2}$ Department of \\ Urban and Rural Planning, School of Architecture and Design, Southwest Jiaotong University, Chengdu, China, ${ }^{3}$ School of \\ Architecture and Urban Planning, Shenzhen University, Shenzhen, China
}

Keywords: COVID-19, public health, well-being, healthy cities, health inequities

\author{
Editorial on Research Topic
}

Supporting Wellbeing During and After COVID-19 in Cities

\section{INTRODUCTION}

This special section of Frontiers In Sustainable Cities, "Supporting Well-being During and After COVID-19 in Cities," represents a collection of articles which answer a key question: how cities can support well-being during and after the COVID-19 pandemic and achieve resilience building and recovery? The outbreak of the COVID-19 pandemic not only threatens public health worldwide but also poses new challenges to urban planning and governance.

Therefore, it is indispensable and urgent to call for attention to such an issue and bring together the wisdom of the academic world to respond to it. This special section provides an exploration of a novel lens through which to understand how cities can shape individuals' health outcomes during COVID-19 in different countries. We aim to address theoretical, methodological, and empirical issues in urban health-related studies during the COVID-19 pandemic.

Edited and reviewed by: Sotiris Vardoulakis, Australian National University, Australia

*Correspondence: Ruoyu Wang

r.wang-54@sms.ed.ac.uk

Specialty section:

This article was submitted to

Health and Cities,

a section of the journal

Frontiers in Sustainable Cities

Received: 19 October 2021 Accepted: 01 November 2021 Published: 23 November 2021

Citation:

Wang $R$, Yang $L$ and Chen $H$ (2021) Editorial: Supporting Wellbeing During and After COVID-19 in Cities.

Front. Sustain. Cities 3:798063. doi: 10.3389/frsc.2021.798063

\section{BACKGROUND}

Throughout human history, pandemics have again and again reshaped the city environment and people's behaviors (Esparza, 2020). For example, the cholera outbreaks in London revealed the link between the built environment and infectious diseases, which later prompted the government to change its urban planning scheme (Johnson, 2006). Therefore, it is essential to address public health issues through urban planning and governance. Existing literature has highlighted five major topics (health inequalities, social environment, natural environment, built environment, and health-related technologies) regarding the city environment and people's well-being during the pandemic (Ahmed et al., 2020; Drefahl et al., 2020; Jaiswal et al., 2020; Lai et al., 2020; Van Dorn et al., 2020; Frumkin, 2021). First, due to the lack of health-related resources, socioeconomically disadvantaged groups are more vulnerable during the pandemic, so whether the pandemic has exacerbated health inequalities and how to prevent the rising of the inequalities have become important issues (Ahmed et al., 2020; Van Dorn et al., 2020). Second, the social environment, such as social capital, has long been recognized as a protective factor for well-being, so how the social environment has changed during the pandemic and how such changes affect well-being are also important issues (Drefahl et al., 2020). Third, the natural environment, such as green space, can help people reduce stress and benefit their health (Wang et al., 2021). Therefore, how people's use of the natural environment, attitudes toward the natural environment have changed during the pandemic, and how these changes influence well-being also attract great attention (Lai et al., 2020). 
Fourth, the built environment, such as design and density, plays an important role in people's daily life (Yang et al., 2021), so it is vital to identify built environment features that affect the risk of COVID-19 (Frumkin, 2021). Last, health-related technologies such as wearable devices can help researchers better understand human behaviors, so the application of new city technologies in COVID-19 prevention has become another major Research Topic (Jaiswal et al., 2020).

In addition, there are significant differences in the ways and capabilities of different countries to cope with the COVID-19 pandemic. However, most of the findings are still from developed countries, while much less attention has been paid to developing countries where people are even more vulnerable during the pandemic. Also, the majority of existing knowledge for COVID19 is mainly from clinical research, while scant attention has been paid to urban studies. Therefore, not only do we need a thorough and scientific understanding of the association between city environment and people's well-being during the pandemic, but this knowledge should also serve as a foundation for the practical/policy implication of alternative futures. In this special session, we want to systematically discuss how cities can better support well-being during and after COVID-19.

\section{SNAPSHOT OF THIS SPECIAL SECTION}

This special section contains four related papers, with two from high income countries (USA and Sweden), and the other two from middle income country (China) as well as low income country (Nigeria) respectively. Specifically, Wortzel et al. investigated the association between green space exposure and mental health in a large sample during the pandemic in the United States. Mean tree canopy density was taken as the green space metric, while mental health was assessed based on COVID19-related worries, anxiety symptoms, depression symptoms, and a composite mental health score of all the above parameters.

\section{REFERENCES}

Ahmed, F., Ahmed, N. E., Pissarides, C., and Stiglitz, J. (2020). Why inequality could spread COVID-19. Lancet Public Health 5:e240. doi: 10.1016/S2468-2667(20)30085-2

Drefahl, S., Wallace, M., Mussino, E., Aradhya, S., Kolk, M., Brandén, M., et al. (2020). A population-based cohort study of socio-demographic risk factors for COVID-19 deaths in Sweden. Nat. Commun. 11, 1-7. doi: 10.1038/s41467-020-18926-3

Esparza, J. (2020). Lessons from history: what can we learn from 300 years of pandemic flu that could inform the response to COVID-19? Am. J. Public Health 110, 1160-1161. doi: 10.2105/AJPH.2020.305761

Frumkin, H. (2021). COVID-19, the built environment, and health. Environ. Health Persp. 129:075001. doi: 10.1289/EHP 8888

Jaiswal, R., Agarwal, A., and Negi, R. (2020). Smart solution for reducing the COVID-19 risk using smart city technology. IET Smart Cities 2, 82-88. doi: 10.1049/iet-smc.2020. 0043

Johnson, S. (2006). The Ghost Map: The Story of London's Deadliest Epidemic and How it Changed the Way we Think About Disease, Cities, Science, and the Modern World. New York, NY: Penguin Books.
The results indicated that green space exposure is beneficial for mental health during the pandemic. Zetterberg et al. used a telephone survey in Sweden during the pandemic to examine the changes in social interactions, emotional support, and instrumental support during the pandemic, as well as how these changes affect self-rated health during the pandemic. The results showed that people living in a neighborhood with higher social capital report more increase in social interaction, instrumental support, and better self-rated health during the pandemic. Song et al. applied semi-structured interviews in Changchun, China, to explore the implications of COVID-19 on spatial planning, wellbeing, and behavioral change. The results suggested that working from home during the pandemic has changed people's way of life and subjective well-being, which poses new challenges for the current spatial planning system in China (e.g., more demand for open public spaces and supportive infrastructure). Otu et al. introduced the early experience of developing and deploying the training application (App) for COVID-19 health workers in Nigeria. The App provides front-line health workers with information about COVID-19 and teaches them how to identify, screen, and manage COVID-19 suspects. App users report a high level of satisfaction with the App, and their knowledge of COVID-19 significantly improves after being trained by the App.

The four included papers can be regarded as an early exploration of how pandemic affects people's life within the city. Generally, pre-pandemic knowledge regarding the association between city environment and people's well-being is still valid during the pandemic. However, we expect more extensive and indepth studies on this topic in the near future to further validate or refute our findings from this collection.

\section{AUTHOR CONTRIBUTIONS}

All authors listed have made a substantial, direct and intellectual contribution to the work, and approved it for publication.

Lai, K. Y., Webster, C., Kumari, S., and Sarkar, C. (2020) The nature of cities and the Covid-19 pandemic. Curr. Opin. Environ. Sustain. 46, 27-31. doi: 10.1016/j.cosust.2020.0 8.008

Van Dorn, A., Cooney, R. E., and Sabin, M. L. (2020). COVID-19 exacerbating inequalities in the US. Lancet 395:1243. doi: 10.1016/S0140-6736(20)30 893-X

Wang, R., Feng, Z., Pearce, J., Zhou, S., Zhang, L., and Liu, Y. (2021). Dynamic greenspace exposure and residents' mental health in Guangzhou, China: From over-head to eye-level perspective, from quantity to quality. Landscape Urban Planning 215:104230. doi: 10.1016/j.landurbplan.2021.10 4230

Yang, L., Ao, Y., Ke, J., Lu, Y., and Liang, Y. (2021). To walk or not to walk? Examining non-linear effects of streetscape greenery on walking propensity of older adults. J. Transport Geogr. 94:103099. doi: 10.1016/j.jtrangeo.2021.10 3099

Author Disclaimer: All claims expressed in this article are solely those of the authors and do not necessarily represent those of their affiliated organizations, or those of the publisher, the editors and the reviewers. Any product that may be evaluated in this article or claim that may be made by its manufacturer is not guaranteed or endorsed by the publisher. 
Conflict of Interest: The authors declare that the research was conducted in the absence of any commercial or financial relationships that could be construed as a potential conflict of interest.

Publisher's Note: All claims expressed in this article are solely those of the authors and do not necessarily represent those of their affiliated organizations, or those of the publisher, the editors and the reviewers. Any product that may be evaluated in this article, or claim that may be made by its manufacturer, is not guaranteed or endorsed by the publisher.

Copyright (c) 2021 Wang, Yang and Chen. This is an open-access article distributed under the terms of the Creative Commons Attribution License (CC BY). The use, distribution or reproduction in other forums is permitted, provided the original author(s) and the copyright owner(s) are credited and that the original publication in this journal is cited, in accordance with accepted academic practice. No use, distribution or reproduction is permitted which does not comply with these terms. 